\title{
INDEX OF SPECIES
}

Page numbers in italics refer to the main subjects of the papers

Adalia bipunctata, 227-232

decempunctata, 227-232

Aegilops speltoides, 73-83 longissima, 73-83

Agrostis tenuis, 142, 385

Andropogon, 144

Anthoxanthum odoratum, 142

Arabidopsis thaliana, 342

Aspergillus indulans, 260

Avena caudii, 144

fatua, 142

Backrium subtilis, 255

Biston betularia, 227-232

Bromus mollis, 141

Campelia zanonia, 253

Carrot, 97-109

Centaurea sp. 144 procurrens, 144

Cepaea nemoralis, 127-139

horteusis, 129, 397-398

Chlamydomonas reinhardi, 257

Clarkia speciosa, 253

Coleus, 248

Collinsia heterophilla, 141

Crepis capillaris, 233

Drosophila sp., $211-225,247,257,316,351$ melanogarter, 27-32, 211, 289-301, 327 pseudo-obscura, 90, 366

Eragrostis bipennata, 144

Escherichia coli, 255, 261

Flax, 63-72, 327-340

Fomes cajanderi, 15

Gasterosteus aculeatus, 273-287, 303-312

Haemanthus, 252

Helix aspersa, 397 pomatia, 397

Ictalurus, 274
Klebsiella aerogenes, 261

Lepomis, 274

Limnaea peragra, 224, 397

Linum sp., 369-379

Lotus corniculatus, 241, 381-386

Megadyptes antipodes, 314

Melanoplus, 233

Melospiza melodia, 315

Microtus ochrogaster, 53-62

plunsylvanicus, 53-62

Mouse, 262

Neurospora crassa, 260, 261

Nicotiana rustica, 111-126, 342, 387-395

Panaxia dominula, 384

Papaver dubium, 177-187, 189-197 rhoeas, 196

Partula suturalis, 397

Parus major, 313-325

Pelargonium, 1-13

Peromyscus maniculatus bairdii, 315

Phigalia pedaria, 227-232

Plantago lanceolata, 169-176

Platichthys flesus, 244-245

Pleuronectes platessa, 244-245

Polyporus betulinus, 15 palutris, 15

Potentilla glandulosa, 384

Quercus cerris, 228

ithaburensis, 144

petraea, 228

robor, 228

Rye, 233-240

Saccharomyces cerevisiae, 258, 259, 260, 265-271

Schizophyllum commune, 15-26, 33-40, 112, 341 349

Tradescantia paludosa, 233

Trifolium repens 241-245 
Triticum aestivum, 258 diccocoides, 144 longissimum, 73-83, 141-167 speltoides, 73-83, 141-167

Triturus, 256

Troglodytes aëdon, 315

Ustilago maydis, 257
Vicia sativa, 383

Vole, 53-62

Wheat, 73-83, 141-167, 258

Xenopus, 256

Zea mays, 252 